\title{
A Simple Nomogram to Predict Contrast-Induced Acute Kidney Injury in Patients with Congestive Heart Failure Undergoing Coronary Angiography
}

\author{
Li Lei $\triangle,{ }^{1,2}$ Yibo He, ${ }^{2}$ Zhaodong Guo, ${ }^{2}$ Bowen Liu, ${ }^{3}$ Jin Liu, ${ }^{2}$ Zhiqiang Nie, ${ }^{2}$ \\ Guanzhong Chen $\mathbb{D}^{3},{ }^{3}$ Liwei Liu, ${ }^{1,2}$ Mengfei Lin, ${ }^{4}$ Wenhe Yan, ${ }^{4}$ Shiqun Chen $\mathbb{D},{ }^{2}$ \\ Chen Jiyan $\left(D,{ }^{1,2}\right.$ and Yong Liu ${ }^{1,2}$ \\ ${ }^{1}$ The Second School of Clinical Medicine, Southern Medical University, Guangzhou, Guangdong, China \\ ${ }^{2}$ Department of Cardiology, Provincial Key Laboratory of Coronary Heart Disease, Guangdong Cardiovascular Institute, \\ Guangdong Provincial People's Hospital Affiliated with South China University of Technology, \\ Guangdong Academy of Medical Sciences, Guangzhou, Guangdong, China \\ ${ }^{3}$ Guangdong Provincial People's Hospital, School of Medicine, South China University of Technology, Guangzhou, \\ Guangdong, China \\ ${ }^{4}$ Department of Cardiology, Maoming People's Hospital, Maoming, Guangdong, China
}

Correspondence should be addressed to Chen Jiyan; chenjiyandr@126.com and Yong Liu; liuyong@gdph.org.cn

Received 22 June 2020; Revised 12 February 2021; Accepted 10 March 2021; Published 23 March 2021

Academic Editor: Robert Chen

Copyright (c) 2021 Li Lei et al. This is an open access article distributed under the Creative Commons Attribution License, which permits unrestricted use, distribution, and reproduction in any medium, provided the original work is properly cited.

Background. Patients with congestive heart failure (CHF) are vulnerable to contrast-induced kidney injury (CI-AKI), but few prediction models are currently available. Therefore, we aimed to establish a simple nomogram for CI-AKI risk assessment for patients with CHF undergoing coronary angiography. Methods. A total of 1876 consecutive patients with CHF (defined as New York Heart Association functional class II-IV or Killip class II-IV) were enrolled and randomly (2:1) assigned to a development cohort and a validation cohort. The endpoint was CI-AKI defined as serum creatinine elevation of $\geq 0.3 \mathrm{mg} / \mathrm{dL}$ or $50 \%$ from baseline within the first 48-72 hours following the procedure. Predictors for the simple nomogram were selected by multivariable logistic regression with a stepwise approach. The discriminative power was assessed using the area under the receiver operating characteristic (ROC) curve and was compared with the classic Mehran score in the validation cohort. Calibration was assessed using the Hosmer-Lemeshow test and 1000 bootstrap samples. Results. The incidence of CI-AKI was 9.06\% (170) in the total sample, $8.64 \%(n=109)$ in the development cohort, and $9.92 \%(n=61)$ in the validation cohort $(P=0.367)$. The simple nomogram including four predictors (age, intra-aortic balloon pump, acute myocardial infarction, and chronic kidney disease) demonstrated a similar predictive power as the Mehran score (area under the curve: $0.80 \mathrm{vs.} 0.75, P=0.061$ ), as well as a well-fitted calibration curve. Conclusions. The present simple nomogram including four predictors is a simple and reliable tool to identify $\mathrm{CHF}$ patients at risk of CI-AKI, whereas further external validations are needed.

\section{Introduction}

Contrast-induced kidney injury (CI-AKI) is a common complication following coronary angiography (CAG) or percutaneous coronary intervention (PCI), and the occurrence of CI-AKI has been demonstrated to be related to poor outcomes [1-3]. Published guidelines recommend hydration therapy during the perioperative period of coronary catherization as one of the important strategies to prevent CI-AKI $[4,5]$. However, for patients with congestive heart failure (CHF), the extra fluid load might lead to further adverse events of acute heart failure [6, 7], even though hydration volume is halved in such patients. Therefore, precise stratification and identification of patients at high risk of CI-AKI are required to perform preventive hydration accurately to reduce unnecessary overload complications. 
Previous studies have reported various prediction models for CI-AKI, but few of them were developed for CHF patients specifically, even though CHF patients are vulnerable to CIAKI [8]. In addition, the classic Mehran score, which includes 8 variables, might be too complicated for clinical application [9]. Therefore, in this study, we aimed to establish a simple nomogram for CI-AKI risk assessment among patients with CHF undergoing CAG/PCI.

\section{Materials and Methods}

2.1. Patients. The current study population was based on a prospective observation cohort (PREdictive Value of COntrast voluMe to creatinINe Clearance Ratio, PRECOMIN, NCT01400295) which enrolled consecutive patients undergoing CAG/PCI in Guangdong Provincial People's Hospital between January 2010 and October 2012. In this study, patients with CHF (defined as New York Heart Association (NYHA) functional class II-IV or Killip class IIIV) undergoing CAG/PCI were enrolled. [10] The exclusion criteria were pregnancy, lactation, contrast exposure within the 7 days before or 3 days after the procedure, cardiovascular surgery, no use of low-osmolarity contrast agents, undergoing hemodialysis, missing preoperative or postoperative creatinine, malignancy, and no use of isotonic saline for hydration). The Ethics Committee of the Guangdong Provincial People's Hospital approved this study. All the patients involved provided written informed consent and were randomly assigned to a development cohort and a validation cohort in a 2:1 ratio.

\subsection{Coronary Angiography and Laboratory Examination.} The procedures were conducted by interventional cardiologists following published guidelines, institutional policy, and clinical routine. [11] Patients undergoing a nonemergency procedure received hydration therapy $(0.5-1 \mathrm{~mL} /$ $\mathrm{kg} / \mathrm{h}$ for at least 2-12 hours before and 6-24 hours after the procedure). Patients undergoing emergency procedures received unspecified hydration therapy before the procedure. Serum creatinine (SCr) was measured for all patients at admission and at 1,2, and 3 days after the procedure using the Jaffe method.

2.3. Endpoint and Definitions. The endpoint of this study was CI-AKI defined as a SCr elevation of $\geq 0.3 \mathrm{mg} / \mathrm{dL}$ or $50 \%$ from baseline within the first 48 to 72 hours following contrast exposure. [12] Baseline characteristics, angiographic data, and medications were prospectively defined and have been reported in a previous study [13]. The definitions of hypotension, diabetes, anemia, chronic kidney disease (CKD), and intra-aortic balloon pump (IABP) were the same as those used for the Mehran score [9]. Acute myocardial infarction (AMI) was defined according to the third universal definition of myocardial infarction [14]. Hypoalbuminemia was defined as serum albumin $<35 \mathrm{~g} / \mathrm{L}$ [15]. Age, weight, heart rate (HR), and contrast volume were defined as continuous variables. Angiotensin-converting enzyme inhibitors (ACEIs)/angiotensin-receptor blockers
(ARBs), diuretics, and beta blockers were defined as the prescription of these medicines during the perioperative period. To calculate the estimated glomerular filtration rate (eGFR), the Modification of Diet in Renal Disease (MDRD) equation was used: eGFR $\left(\mathrm{mL} / \mathrm{min} / 1.73 \mathrm{~m}^{2}\right)=186 *\left(\mathrm{SCr}^{\wedge}\right.$ $-1.154) *\left(\right.$ Age $\left.^{\wedge}-0.203\right) * 0.742$ (if female). The unit of $\mathrm{SCr}$ in this formula is $\mathrm{mg} / \mathrm{dl}$. CKD was defined as eGFR $<60 \mathrm{~mL} / \mathrm{min} / 1.73 \mathrm{~m}^{2}$. Patients were further divided into 5 CKD stages according to the guidelines (CKD G1: eGFR $\geq 90 \mathrm{~mL} / \mathrm{min} / 1.73 \mathrm{~m}^{2}$; CKD G2: eGFR: $60-90 \mathrm{~mL} /$ $\mathrm{min} / 1.73 \mathrm{~m}^{2}$; CKD G3: eGFR: $30-60 \mathrm{~mL} / \mathrm{min} / 1.73 \mathrm{~m}^{2}$; CKD G4: eGFR: $15-30 \mathrm{~mL} / \mathrm{min} / 1.73 \mathrm{~m}^{2}$; and CKD G5: eGFR: $<15 \mathrm{~mL} / \mathrm{min} / 1.73 \mathrm{~m}^{2}$ ). [16] And they were also divided into 3 categories according to their heart function based on NYHA or Killip class. All eligible patients enrolled were followed up at 1 month, 6 months, and every 1 year after enrollment until April 2019.

2.4. Statistical Analysis. All the patients involved were randomly assigned to a development cohort and a validation cohort in a 2:1 ratio. Continuous variables were compared with an unpaired, 2-tailed $t$-test and are expressed as the mean \pm SD or were compared through the Wilcoxon ranksum test and are expressed as the median \pm interquartile. Categorical variables were compared using the $\chi^{2}$ test or Fisher's exact test and are expressed as percentages. Kaplan-Meier curve and multivariable Cox proportional hazard regression adjusted for known risk factors in the context of long-term prognosis were performed to explore the association between CI-AKI and long-term mortality. $[17,18]$.

To build the nomogram, candidate variables that were imbalanced between groups in the development cohort or that are clinically important, such as risk factors included in the traditional Mehran score, were included in the univariable logistic analysis. Variables with $>15 \%$ missing values were not considered candidates, i.e., low-density lipoprotein-C and $\mathrm{HbAlc}$. Significant variables from the univariable logistic analysis were then included in the multivariable logistic analysis. A backward stepwise approach was performed to screen the variables by successively removal of nonsignificant $(P<0.1)$ covariates until all the remaining variables were statistically significant. Then, we manually investigated the contribution of the remaining variables to determine the final predictors. Collinearity between variables was also evaluated. A nomogram was then formulated based on the results and by using the rms package of $\mathrm{R}$. To form the nomogram, each regression coefficient in the multivariate logistic regression was proportionally converted into a 0 - to 100 -point scale. The variable with the highest $\beta$ coefficient (absolute value) was assigned 100 points. The points are added across each variable to calculate the total points, which are finally converted to predicted probabilities. The performance of the nomogram was assessed using the area under the receiver operating characteristic (ROC) curve and concordance C-statistic for discriminative ability. For validation, a bootstrap method (1000 times) was performed in both the 
TABLE 1: Baseline characteristics in the development cohort.

\begin{tabular}{|c|c|c|c|c|}
\hline Variables & Missing value, $n(\%)$ & CI-AKI $(n=109)$ & Non-CI-AKI $(n=1152)$ & $P$ value \\
\hline Age, years & $0(0)$ & $69.72 \pm 10.75$ & $64.24 \pm 10.36$ & $<0.001$ \\
\hline Age $\geq 65$ years, $n(\%)$ & $0(0)$ & $80(73.39)$ & $584(50.69)$ & $<0.001$ \\
\hline Age $\geq 75$ years, $n(\%)$ & $0(0)$ & $41(37.61)$ & $203(17.62)$ & $<0.001$ \\
\hline Female sex, $n(\%)$ & $0(0)$ & $33(30.28)$ & $281(24.39)$ & 0.175 \\
\hline Weight, kg & $8(0.63)$ & $62.46 \pm 9.84$ & $64.70 \pm 10.93$ & 0.027 \\
\hline SBP, mmHg & $3(0.24)$ & $128.83 \pm 28.19$ & $130.77 \pm 20.39$ & 0.487 \\
\hline $\mathrm{DBP}, \mathrm{mmHg}$ & $4(0.32)$ & $73.64 \pm 12.70$ & $76.54 \pm 11.98$ & 0.024 \\
\hline $\mathrm{HR}, \mathrm{bpm}$ & $3(0.24)$ & $80.04 \pm 16.94$ & $74.86 \pm 13.72$ & 0.003 \\
\hline \multicolumn{5}{|l|}{ Medical history } \\
\hline Hypotension, $n(\%)$ & $4(0.32)$ & $11(10.28)$ & $25(2.17)$ & $<0.001$ \\
\hline $\mathrm{CKD}, n(\%)$ & $0(0)$ & $57(52.29)$ & $236(20.49)$ & $<0.001$ \\
\hline CKD stages & $0(0)$ & & & $<0.001$ \\
\hline CKD G1, $n(\%)$ & & $14(12.84)$ & $358(31.08)$ & \\
\hline CKD G2, $n(\%)$ & & $38(34.86)$ & $558(48.44)$ & \\
\hline CKD G3, $n(\%)$ & & $41(37.61)$ & $221(19.18)$ & \\
\hline CKD G4, $n(\%)$ & & $16(14.68)$ & $13(1.13)$ & \\
\hline CKD G5, $n(\%)$ & & $0(0)$ & $2(0.17)$ & \\
\hline LVEF, \% & $137(10.86)$ & $50.36 \pm 12.54$ & $57.50 \pm 13.15$ & $<0.001$ \\
\hline LVEF < 40\%, $n(\%)$ & $137(10.86)$ & $20(19.80)$ & $123(12.02)$ & 0.025 \\
\hline Heart function (NYHA class) & $0(0)$ & & & $<0.001$ \\
\hline II, $n(\%)$ & & $28(25.69)$ & $742(64.41)$ & \\
\hline III, $n(\%)$ & & $14(12.84)$ & $98(8.51)$ & \\
\hline $\mathrm{IV}, n(\%)$ & & $3(2.75)$ & $15(1.30)$ & \\
\hline Heart function (Killip class) & $0(0)$ & & & $<0.001$ \\
\hline II, $n(\%)$ & & $42(38.53)$ & $250(21.70)$ & \\
\hline III, $n(\%)$ & & $10(9.17)$ & $34(2.95)$ & \\
\hline $\mathrm{IV}, n(\%)$ & & $12(11.01)$ & $13(1.13)$ & \\
\hline Hypertension, $n(\%)$ & $1(0.08)$ & 85 (77.98) & $703(61.08)$ & $<0.001$ \\
\hline Hyperlipidemia, $n(\%)$ & $0(0)$ & $16(14.68)$ & $166(14.41)$ & 0.939 \\
\hline Hypoalbuminemia, $n$ (\%) & $108(8.56)$ & $60(68.18)$ & $479(44.98)$ & $<0.001$ \\
\hline Anemia, $n(\%)$ & $21(1.67)$ & $54(50.47)$ & $378(33.36)$ & $<0.001$ \\
\hline AMI, $n(\%)$ & $4(0.32)$ & $64(58.72)$ & $297(25.87)$ & $<0.001$ \\
\hline Diabetes, $n(\%)$ & $0(0)$ & $38(34.86)$ & $293(25.43)$ & 0.032 \\
\hline $\mathrm{CAD}, n(\%)$ & $6(0.48)$ & $104(95.41)$ & $1068(93.19)$ & 0.373 \\
\hline \multicolumn{5}{|l|}{ Laboratory examination } \\
\hline LDL-C, $\mathrm{mmol} / \mathrm{L}$ & $224(17.76)$ & $2.94 \pm 1.10$ & $2.67 \pm 0.95$ & 0.045 \\
\hline HDL-C, mmol/L & $224(17.76)$ & $0.96 \pm 0.30$ & $1.05 \pm 2.20$ & 0.267 \\
\hline $\mathrm{SCr}, \mu \mathrm{mol} / \mathrm{L}$ & $0(0)$ & $120.76 \pm 55.33$ & $93.12 \pm 44.14$ & $<0.001$ \\
\hline $\mathrm{eGFR}, \mathrm{ml} / \mathrm{min} / 1.73 \mathrm{~mm}^{2}$ & $0(0)$ & $63.16 \pm 34.49$ & $78.88 \pm 23.74$ & $<0.001$ \\
\hline Hemoglobin, g/L & $73(5.79)$ & $122.19 \pm 21.61$ & $133.06 \pm 16.42$ & $<0.001$ \\
\hline HbAlc, \% & $270(21.41)$ & $6.97 \pm 1.59$ & $6.59 \pm 1.36$ & 0.040 \\
\hline \multicolumn{5}{|l|}{ Medications } \\
\hline ACEI/ARB, $n(\%)$ & $0(0)$ & $87(79.82)$ & $1004(87.15)$ & 0.032 \\
\hline Beta blocker, $n(\%)$ & $1(0.08)$ & $68(62.39)$ & $992(86.19)$ & $<0.001$ \\
\hline Statin, $n(\%)$ & $0(0)$ & $102(93.58)$ & $1113(96.61)$ & 0.109 \\
\hline Diuretics, $n(\%)$ & $1(0.08)$ & $52(47.71)$ & $236(20.50)$ & $<0.001$ \\
\hline \multicolumn{5}{|l|}{ Procedure } \\
\hline PCI, $n(\%)$ & $61(4.84)$ & $71(78.02)$ & $782(70.51)$ & 0.129 \\
\hline Hydration volume, $\mathrm{mL}$ & $32(1.71)$ & $1130.31 \pm 674.82$ & $788.37 \pm 433.57$ & $<0.001$ \\
\hline Contrast volume, $\mathrm{mL}$ & $0(0)$ & $139.72 \pm 72.85$ & $133.88 \pm 67.78$ & 0.422 \\
\hline Contrast volume $\geq 100 \mathrm{~mL}, n(\%)$ & $0(0)$ & $81(74.31)$ & $869(74.43)$ & 0.795 \\
\hline Contrast volume $\geq 200 \mathrm{~mL}, n(\%)$ & $0(0)$ & $21(19.27)$ & $224(19.44)$ & 0.964 \\
\hline Mehran score & $23(1.82)$ & $9.75 \pm 6.19$ & $4.72 \pm 4.18$ & $<0.001$ \\
\hline Peri-procedure IABP, $n(\%)$ & $0(0)$ & $26(23.85)$ & $35(3.04)$ & $<0.001$ \\
\hline
\end{tabular}

CI-AKI: contrast-induced acute kidney injury; SBP: systolic blood pressure; DBP: diastolic blood pressure; HR: heart rate; LVEF: left ventricular ejection fraction; CKD: chronic kidney disease; NYHA: New York Heart Association; AMI: acute myocardial infarction; CAD: coronary artery disease; LDL-C: lowdensity lipoprotein-C; HDL-C: high-density lipoprotein-C; SCr: serum creatinine; eGFR: estimate glomerular filtration rate; ACEI: angiotensin-converting enzymes inhibitors; ARB: angiotensin-receptor blockers; PCI: percutaneous coronary intervention; and IABP: intra-aortic balloon pump. 
TABLE 2: Univariable logistic regression analysis of CI-AKI.

\begin{tabular}{|c|c|c|}
\hline Variable & OR $(95 \% \mathrm{CI})$ & $P$ value \\
\hline Age, years & $1.06(1.04-1.08)$ & $<0.001$ \\
\hline Age $\geq 65$ years vs. age $<65$ years & $2.68(1.73-4.17)$ & $<0.001$ \\
\hline Age $\geq 75$ years vs. age $<75$ years & $2.82(1.86-4.27)$ & $<0.001$ \\
\hline Weight, kg & $0.98(0.96-1.00)$ & 0.040 \\
\hline $\mathrm{HR}, \mathrm{bpm}$ & $1.02(1.01-1.04)$ & $<0.001$ \\
\hline $\mathrm{DBP}, \mathrm{mmHg}$ & $0.98(0.96-1.00)$ & 0.017 \\
\hline CKD vs. no CKD & $4.25(2.85-6.36)$ & $<0.001$ \\
\hline $\mathrm{SCr}, \mu \mathrm{mol} / \mathrm{L}$ & $1.01(1.00-1.01)$ & $<0.001$ \\
\hline $\mathrm{eGFR}, \mathrm{ml} / \mathrm{min} / 1.73 \mathrm{~mm}^{2}$ & $0.97(0.96-0.98)$ & $<0.001$ \\
\hline $\mathrm{LVEF}<40 \%$ vs. $\mathrm{LVEF} \geq 40 \%$ & $1.81(1.07-3.05)$ & 0.027 \\
\hline Hypoalbuminemia vs. no hypoalbuminemia & $2.62(1.65-4.17)$ & $<0.001$ \\
\hline Hypertension vs. no hypertension & $2.26(1.41-3.61)$ & $<0.001$ \\
\hline Hypotension vs. no hypotension & $5.16(2.46-10.80)$ & $<0.001$ \\
\hline Anemia vs. no anemia & $2.04(1.37-3.03)$ & $<0.001$ \\
\hline AMI vs. no AMI & $4.08(2.72-6.10)$ & $<0.001$ \\
\hline Diabetes vs. no diabetes & $1.57(1.04-2.38)$ & 0.034 \\
\hline ACEI/ARB vs. no ACEI/ARB & $0.58(0.35-0.96)$ & 0.034 \\
\hline Beta blocker vs. no beta blocker & $0.27(0.17-0.41)$ & $<0.001$ \\
\hline Diuretic vs. no diuretic & $3.54(2.37-5.29)$ & $<0.001$ \\
\hline IABP vs. no IABP & $10.00(5.74-17.40)$ & $<0.001$ \\
\hline PCI vs. no PCI & $1.48(0.89-2.48)$ & 0.131 \\
\hline Hydration volume, $\mathrm{mL}$ & $1.00(1.00-1.00)$ & $<0.001$ \\
\hline Contrast volume, $\mathrm{mL}$ & $1.00(1.00-1.00)$ & 0.392 \\
\hline Contrast volume $\geq 100 \mathrm{~mL}$ vs. contrast volume $<100 \mathrm{~mL}$ & $0.94(0.60-1.48)$ & 0.795 \\
\hline Contrast volume $\geq 200 \mathrm{~mL}$ vs. contrast volume $<200 \mathrm{~mL}$ & $0.99(0.60-1.63)$ & 0.964 \\
\hline
\end{tabular}

CI-AKI: contrast-induced acute kidney injury; HR: heart rate; DBP: diastolic blood pressure; CKD: chronic kidney disease; SCr: serum creatinine; eGFR: estimate glomerular filtration rate; LVEF: left ventricular ejection fraction; AMI: acute myocardial infarction; ACEI: angiotensin-converting enzymes inhibitors; ARB: angiotensin-receptor blockers; PCI: percutaneous coronary intervention; and IABP: intra-aortic balloon pump.

development and validation cohorts to evaluate the stability of the C-statistic. Calibration was assessed using the Hosmer-Lemeshow test and the 1000 bootstrap samples to decrease the overfit bias $[19,20]$. Area under curve (AUC) comparison between the nomogram and the Mehran score was performed using DeLong's test in the validation cohort. Missing data were not imputed. In all analyses, $P<0.05$ was considered statistically significant. All analyses were conducted with $R$ software (version 3.6.2; $R$ Foundation for Statistical Computing, Vienna, Austria) and SPSS (version 26.0).

\section{Results}

3.1. Baseline Characteristics. The details and missing data of the included patients are listed in Supplementary Table 1. Among the included 1876 CHF patients, 1261 and 615 patients were divided into the development and validation cohorts, respectively. In the total cohort, approximately onefourth were female $(25.11 \%)$. The mean age was $64.77 \pm 10.70$ years, and the mean $\mathrm{SCr}$ was $96.86 \pm 50.73 \mu \mathrm{mol} / \mathrm{L}$. No significant differences were identified between the development and validation cohorts, except for the diastolic blood pressure, contrast volume, and the proportion of percutaneous coronary intervention (PCI) during the procedure.

The incidence of CI-AKI was $9.06 \%(n=170)$ in the total sample, $8.64 \%$ (109 patients) in the development cohort, and $9.92 \%$ (61 patients) in the validation cohort $(P=0.367)$. The
Table 3: Multivariable logistic regression analysis of CI-AKI.

\begin{tabular}{lccc}
\hline Variable & $\beta$ & OR $(95 \%$ CI $)$ & $P$ value \\
\hline Age, years & 0.04 & $1.04(1.02-1.06)$ & 0.001 \\
IABP vs. no IABP & 1.38 & $3.97(2.14-7.37)$ & $<0.001$ \\
AMI vs. no AMI & 1.18 & $3.27(2.09-5.10)$ & $<0.001$ \\
CKD vs. no CKD & 1.04 & $2.83(1.80-4.44)$ & $<0.001$ \\
\hline
\end{tabular}

CI-AKI: contrast-induced acute kidney injury; IABP: intra-aortic balloon pump; AMI: acute myocardial infarction; and CKD: chronic kidney disease.

distribution of the SCr value within the time frame (24-72 hours after the procedure) is shown in Supplementary Figure 1. For the development cohort, patients complicated with CI-AKI following CAG tended to be older and had lower left ventricular ejection fraction (LVEF), haemoglobin, and eGFR than those without CI-AKI. Patients with CIAKI were also more likely to have hypotension, diabetes, CKD, AMI, and intra-aortic balloon pump (IABP), and they were less likely to be prescribed ACEI/ARB and beta blockers. No significant difference between groups was identified in contrast volume (Table 1).

3.2. Development and Validation of the CI-AKI-Predicting Nomogram. The results of univariate logistic analysis are detailed in Table 2. Through multivariate logistic analysis and a backward stepwise approach, age (OR: 1.04 95\%, CI: 1.02-1.06), IABP (OR: 3.97 95\%, CI: 2.14-7.37), AMI (OR: 3.27 95\%, CI: 2.09-5.10), and CKD (OR: 2.83 95\%, CI: $1.80-4.44)$ were selected as predictors of CI-AKI (Table 3). 


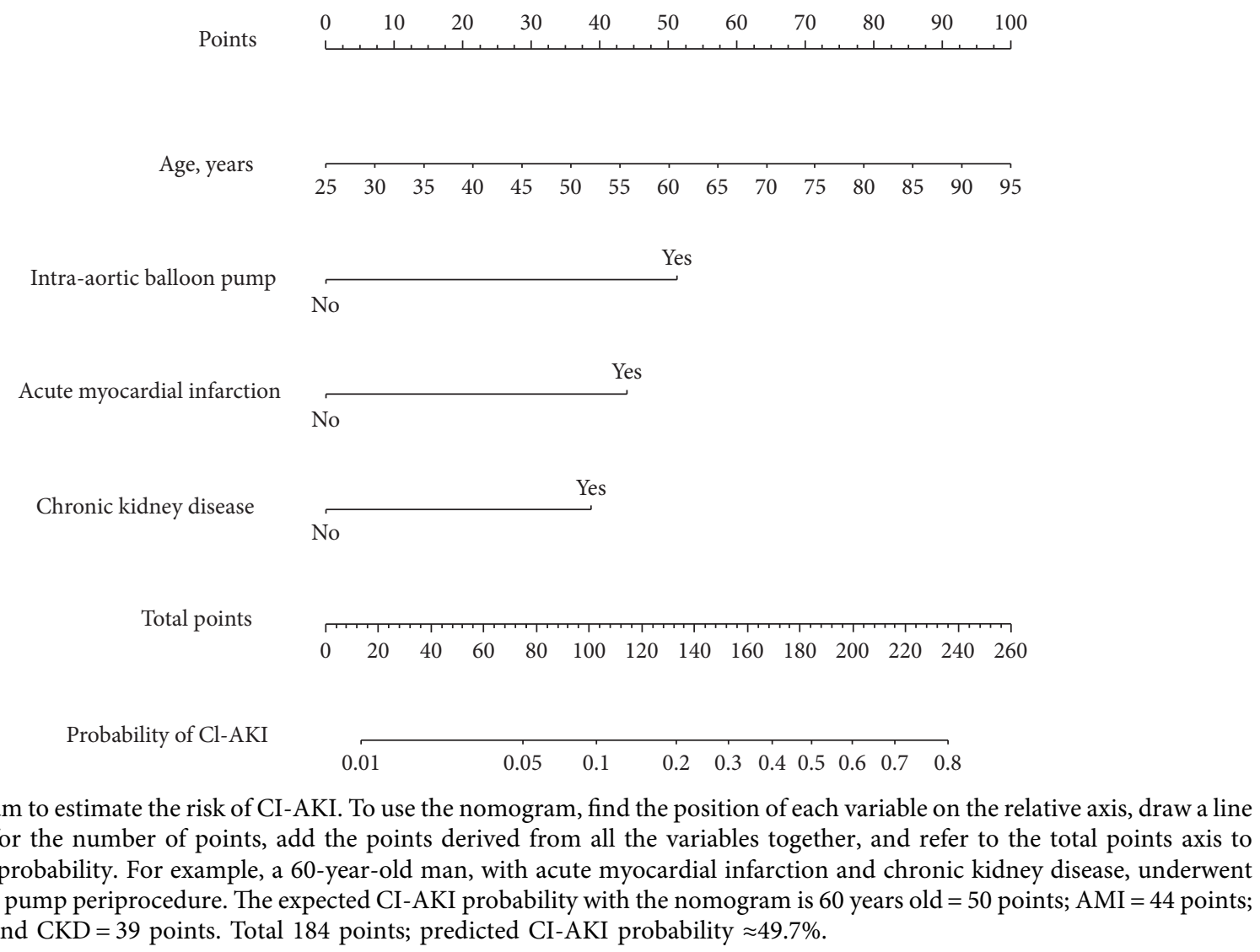

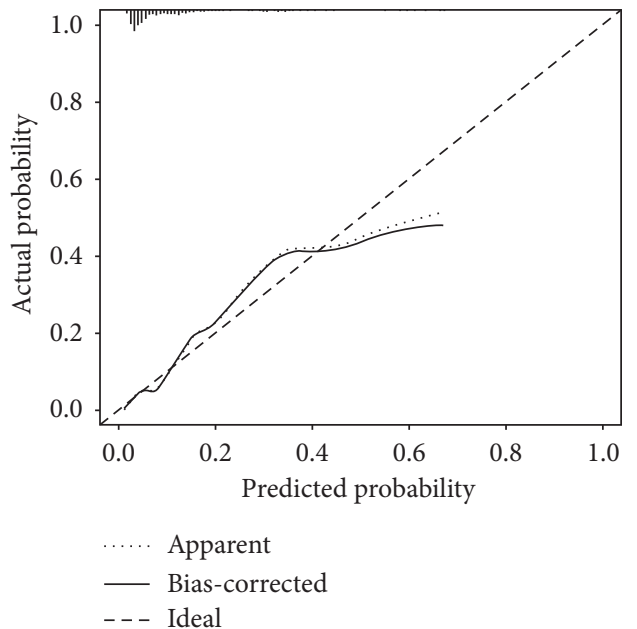

(a)

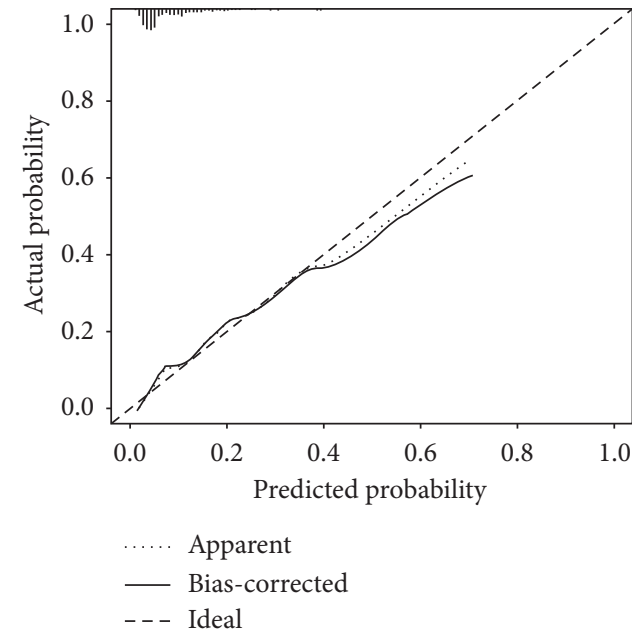

(b)

FIGURE 2: (a) Validity of the predictive value of the nomogram for the estimation of the risk of CI-AKI in the development cohort. (b) Validity of the predictive value of the nomogram for the estimation of the risk of CI-AKI in the validation cohort.

The Hosmer-Lemeshow statistic of multivariable analysis suggested a good fit $\left(\chi^{2}=10.78, P=0.214\right)$.

A simple nomogram based on the selected predictors was formed (Figure 1). The nomogram was internally validated with the bootstrap validation method (1000 times). In the development cohort, the nomogram demonstrated good discriminative power for estimating the risk of CI-AKI, with an unadjusted C-statistic of 0.79 (95\% CI, 0.75-0.84) and a bootstrap-corrected $\mathrm{C}$ statistic of 0.79 . In addition, calibration plots graphically showed good agreement on the presence of CI-AKI between the risk estimation and the observed frequency (Figure 2(a)).

In the validation cohort, the simple nomogram demonstrated a C-statistic of 0.80 (95\% CI 0.75-0.86), which was 


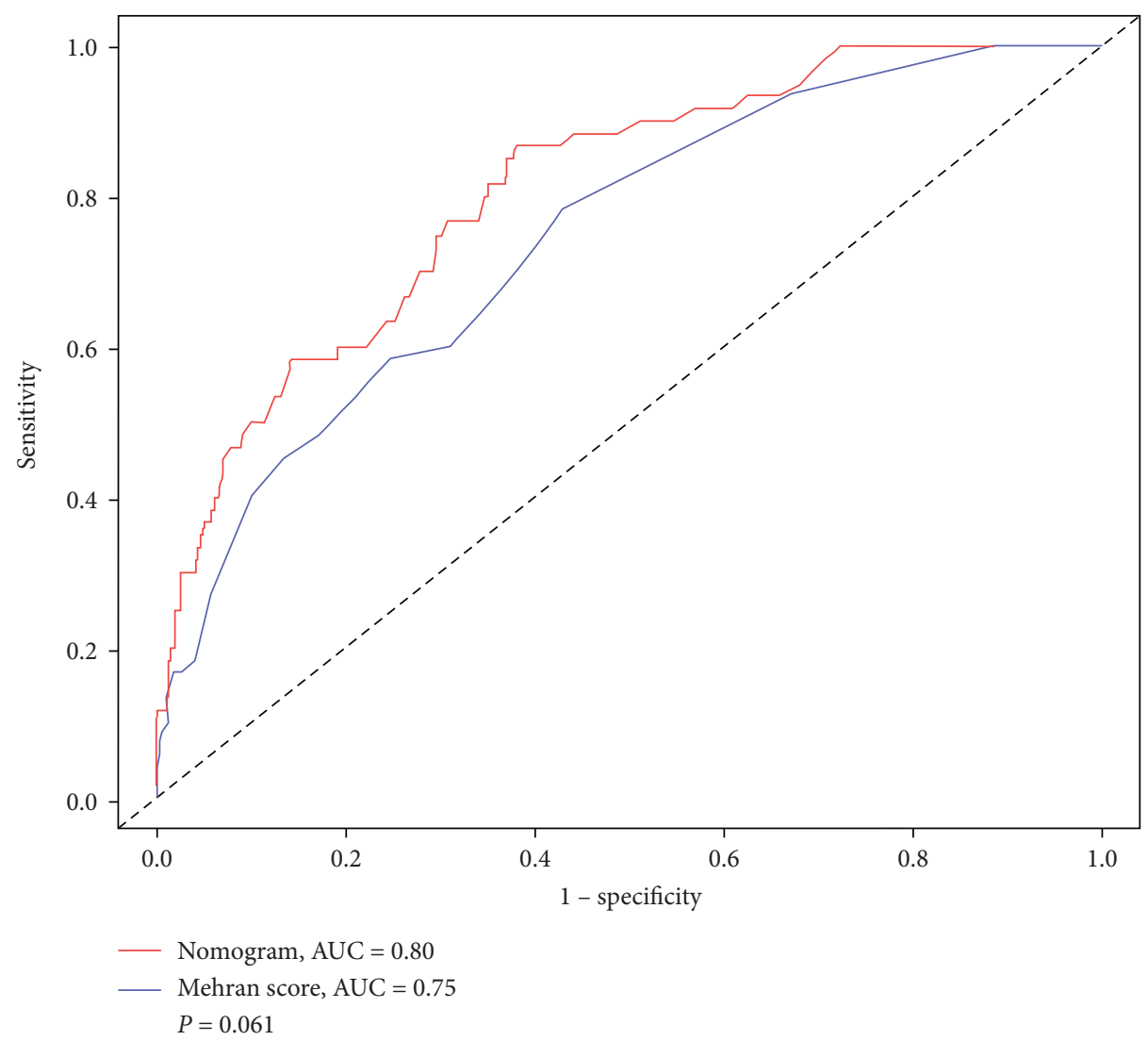

Figure 3: The receiver operator characteristic curves of the nomogram and Mehran score in the validation cohort.

similar to that of the Mehran score (AUC: 0.75, 95\% CI 0.68-0.81) among patients with CHF $(P=0.061$, Figure 3) and a bootstrap-corrected $\mathrm{C}$ statistic of 0.80 . Moreover, there was also a good calibration curve for the risk estimation (Figure 2(b)).

The discriminative ability and calibration were also tested among patients with different stages of CHF and stages of CKD, respectively. No significant difference in discriminative ability was identified, except for the comparison between "Heart function II" and "Heart function IV." Hosmer-Lemeshow statistic suggested a good fit in all stages of CHF and stages of CKD (Supplementary Table 2).

3.3. Risk of CI-AKI Based on the Nomogram Scores. Based on the predicted incidence of CI-AKI in relation to different total nomogram scores, we further divided the patients into 5 score categories: scores $<50 \quad$ (risk $=1.96 \%$ ), $50 \leq$ scores $<100 \quad$ (risk $=4.71 \%), \quad 100 \leq$ scores $<150$ (risk $=13.90 \%), \quad 150 \leq$ scores $<200 \quad$ (risk $=39.09 \%)$, and scores $\geq 200$ (risk $=65.78 \%$ ). The rates of CI-AKI in the validation cohort were close to those in the development cohort inside each of the 5 score categories (Supplementary Figure 2).

The optimal cut-off value of the total nomogram scores to identify patients at risk was determined to be 100 . The sensitivity and specificity used to differentiate the presence from the absence of CI-AKI were $69.7 \%$ and $75.6 \%$ in the development cohort and $70.0 \%$ and $74.5 \%$ in the validation cohort, respectively. The total nomogram scores of 100 to identify patients at risk also demonstrated a good discriminative power in both the development cohort (AUC: 0.73, 95\% CI: $0.68-0.78$, and $P<0.001)$ and the validation cohort (AUC: $0.72,95 \%$ CI: $0.65-0.79$, and $P<0.001$ ).

\section{Discussion}

The present study might be the first to develop a simple nomogram for the prediction of CI-AKI among CHF patients undergoing CAG/PCI. Through multivariable logistic regression analysis and a stepwise approach, a nomogram based on four factors (age, IABP use, AMI, and CKD) was constructed. In particular, the simple nomogram demonstrated a similar discriminative power as the classic Mehran score in the validation cohort, as well as good stability and calibration.

To define CHF patients in this study, two classification methods (NYHA and Killip) were used interchangeably. Previous studies had already taken this method to identify CHF patients [21, 22]. In our clinical routine, we evaluate the heart function of AMI and non-AMI patients with Killip and NYHA system, respectively. Using these two systems interchangeably may help identifying patients with impaired heart function more concisely.

In this study of the CHF population, the overall incidence of CI-AKI was $9.06 \%$. The previously reported 
incidence of CI-AKI has not been consistent among different studies. Among the patients with a low-risk of ordinary coronary artery disease, the CI-AKI incidence was as low as $2-3 \%$, while in the high-risk population, the CI-AKI incidence could be as high as $19 \%$ or $50 \%[2,3]$. Since heart failure patients might be complicated with haemodynamic disturbances, which might further cause kidney dysfunction $[23,24]$, the relatively high incidence of CI-AKI among our $\mathrm{CHF}$ cohort was mostly rational. Moreover, we noticed that some patients were missing $\mathrm{SCr}$ data on day 3, which may lead to the underestimation of the incidence of CI-AKI. However, many previous studies had limited the diagnosis time frame of CI-AKI to 48 hours, or even 24 hours $[9,25]$. In the study conducted by Kim et al., CI-AKI was defined as an increase in $\mathrm{SCr}$ of $\geq 50 \%$ or $0.3 \mathrm{mg} / \mathrm{dL}$, or a decrease in eGFR of $\geq 25 \%$ within 24 hours after PCI [25].

The present simple nomogram was constructed based on age, AMI, IABP, and CKD, which are common risk factors associated with CI-AKI, as reported by numerous previous studies [12, 26, 27]. Thomas T. Tsai et al. conducted a study including 985,737 consecutive patients who underwent PCI at 1,253 sites in America and found that ST-segment elevation myocardial infarction (STEMI) (OR: 2.60; 95\% CI: 2.53-2.67) as well as non-ST-segment elevation myocardial infarction (NSTEMI) (OR: 1.81; 95\% CI: 1.61-2.04) were independently associated with CI-AKI. Additionally, each 10-year increase in age (OR: 1.15; 95\% CI: 1.14-1.16), IABP before the procedure (OR: 2.13; 95\% CI: 1.92-2.35), and CKD stage 3-5 were all risk factors for CI-AKI [2]. Pierre Aubry et al. performed a retrospective cross-sectional population-based study involving 1,047,329 cases of contrast exposure in Europe to quantify the effect of risk factors for CI-AKI. By multivariate analysis, age $>80$ years (OR: 2.7; 95\% CI: 2.6-2.8) and CKD (OR: 2.3; 95\% CI: 2.2-2.3) were identified as independent risk factors for CI-AKI [28]. For patients in Asia, Pei-Chun Fan et al. developed and validated a risk prediction model (ADVANCIS score) for incident CIAKI based on 82,186 patients admitted for acute coronary syndrome (ACS) receiving PCI. Through multivariable logistic regression analysis, age (OR: 1.02; 95\% CI: 1.01-1.02), CKD (OR: 11.38; 95\% CI: 9.86-13.13), and IABP use (OR: 1.80; 95\% CI: $1.62-2.00)$ were strongly associated with the incidence of CI-AKI. [29].

The nomogram developed in this study is a simple but efficient prediction model that includes only four predictors. We noticed that some important risk factors reported by previous results were not included in the current simple nomogram, such as contrast volume and diabetes [30]. The nonsignificant difference between the CI-AKI and non-CIAKI subjects in contrast volume may be one of the reasons. In addition, some recent studies have found that the dose of contrast agent is not necessarily related to the incidence of CI-AKI $[31,32]$. Also, with the development of coronary interventional treatment techniques, the volume of contrast agents is gradually reduced [9]. Moreover, in the current study, all patients included were restricted to use low-osmolarity contrast agents during the procedure, and the risk of kidney impairment was reduced by the usage of lowosmolarity and isotonic contrast agents [33]. The risk of CI-
AKI might be due to haemodynamic instability rather than direct injury by contrast agents. Regarding diabetes mellitus, a long course of hyperglycaemia would result in renal microcirculation disorder and renal dysfunction, which might affect the incidence of CI-AKI $[34,35]$. However, there is no evidence suggesting that short-term hyperglycaemia associated with diabetes mellitus without renal dysfunction would increase the risk of CI-AKI. Therefore, the novel model we developed directly included CKD as one of the predictors rather than diabetes mellitus. Moreover, adding contrast volume and diabetes to the nomogram did not significantly increase the C-statistic (>10\%).

The Mehran score is one of the most classic CI-AKI risk estimating system, which was developed based on 8,357 patients undergoing PCI [9]. Comparing with the Mehran score, the current nomogram has several strengths. First, our previous study showed that the CI-AKI definition of this study has higher population attributable risk (PAR) than that of the CI-AKI definition of the Mehran score, which means the current CI-AKI definition has better prognostic value [36]. Second, the current nomogram with only 4 variables was established for CHF patients specifically. Less variables lead to easier clinical implication. However, the nomogram also has some shortness. First, the nomogram was developed based on a relatively small cohort. Second, the nomogram lacks external validation.

We also noticed that several researches have provided nomograms to predict acute kidney injury (AKI) lately. However, these nomograms were developed for different patients specifically [37-39]. By analysing the Medical Information Mart for Intensive Care- (MIMIC-) III v. 1.4 database, Deng et al. established a nomogram to predict the risk of septic AKI within the first $24 \mathrm{~h}$ after admission of intensive care unit. Among the included 2,917 sepsis patients without $\mathrm{CKD}$, the nomogram with 7 variables demonstrated well-fitted calibration curves and good C-indexes in both the training and validation cohorts [37]. In another study conducted by $\mathrm{Xu}$ et al., they developed a preprocedural nomogram with 5 variables to predict the risk of AKI among patients undergoing nephrectomy [38]. Comparing with these nomograms, our nomogram was developed specifically for the CHF population undergoing CAG/PCI. Moreover, with fewer and readily accessed variables, our nomogram was easier for clinical implication in the department of cardiology.

Overall, in this study, we established a novel CI-AKI risk prediction nomogram specified for the CHF population. In this simple nomogram, patients with more than 100 total points are identified as at-risk patients, accounting for nearly $30 \%$ of the entire CHF cohort. The threshold of 100 points (approximately $10 \%$ risk) was also similar to the risk threshold of previous models $[9,29]$.

4.1. Limitations. Our study had some limitations. First, this study was an analysis of single-centre data and the nomogram we established is not a general one for CI-AKI risk evaluation in any radiological study, but specifically for coronary study/intervention. However, no relevant study 
had so far elucidated the specified risk factors of CI-AKI in CHF patients. Our study provided a new tool for CI-AKI risk assessment among CHF patients, prompting more attention and further studies on risk assessment of patients with comorbidities undergoing coronary catheterization. Second, the present nomogram was not externally validated, although there was a calibration with 1000 bootstrap samples to decrease the overfit bias. Third, our nomogram did not demonstrate better discriminative power than the Mehran score. However, our model has less variables, which makes it easier for clinical implement. Fourth, some other potential risk factors, especially procedural context (i.e., emergency vs. elective procedure), were not included due to the insufficient data. Finally, some patients were discharged within 72 hours after the CAG/PCI, so creatinine levels were not measured on day 3 in these patients, which might lead to the underestimation of the incidence of CI-AKI.

\section{Conclusions}

The presented nomogram with four predictors (age, IABP, $\mathrm{AMI}$, and CKD) is a simple and reliable tool for CI-AKI risk stratification among CHF patients, which enables physicians to identify patients at risk and implement precise prevention strategies in time. However, further external validations are needed before clinical generalization.

\section{Data Availability}

Data relevant to this study are available from the corresponding authors upon reasonable request.

\section{Disclosure}

This article has been presented at the European Society of Cardiology (ESC) Congress 2020 as a conference abstract. The funding body did not participate in the design of the study and collection, analysis, and interpretation of data and in writing the manuscript.

\section{Conflicts of Interest}

The authors declare that they have no conflicts of interest regarding the publication of this paper.

\section{Authors' Contributions}

Li Lei and Yibo He contributed equally to this work. LL, YH, JL, ZN, SC, JC, and YL substantially contributed to the conception and design of the study; LL, YH, ZG, BL, JL, GC, $\mathrm{LL}, \mathrm{ML}$, and WY involved in data collection; LL, YH, JL, ZN, SC, and YL performed data analysis and/or interpretation of data for the work; and LL, YH, YL, and JC drafted the work or revised it critically for important intellectual content; all the authors approved the final version to be published.

\section{Acknowledgments}

The authors thank ESC Congress 2020 for giving them the chance to share their work. This work was supported by the
Beijing Lisheng Cardiovascular Pilot Foundation (Grant no. LHJJ201612127), the Guangdong Provincial People's Hospital Dengfeng Project Fund (Grant no. DFJH201919), the Progress in Science and Technology Project of Guangzhou (Grant no. 201904010470), the Guangdong Provincial People's Hospital Dengfeng Project Fund (Grant no. DFJH2020026), and the China Youth Clinical Research Fund (Grant no. 2017-CCA-VG-020).

\section{Supplementary Materials}

Supplementary Table 1. Baseline characteristics. Supplementary Table 2. C-statistic and Hosmer-Lemeshow statistic of the nomogram in patients with different stages of congestive heart failure and stages of chronic kidney disease. Supplementary Figure 1. The distribution of the serum creatinine value within 24-72 hours after the procedure. Supplementary Figure 2. Risk of contrast-induced acute kidney injury according to the total nomogram score categories. (Supplementary Materials)

\section{References}

[1] E. Seeliger, M. Sendeski, C. S. Rihal, and P. B. Persson, "Contrast-induced kidney injury: mechanisms, risk factors, and prevention," European Heart Journal, vol. 33, no. 16, pp. 2007-2015, 2012.

[2] T. T. Tsai, U. D. Patel, T. I. Chang et al., "Contemporary incidence, predictors, and outcomes of acute kidney injury in patients undergoing percutaneous coronary interventions," JACC: Cardiovascular Interventions, vol. 7, no. 1, pp. 1-9, 2014.

[3] A. Narula, R. Mehran, G. Weisz et al., "Contrast-induced acute kidney injury after primary percutaneous coronary intervention: results from the HORIZONS-AMI substudy," European Heart Journal, vol. 35, no. 23, pp. 1533-1540, 2014.

[4] U. Nyman, J. Ahlkvist, J. Ahlkvist et al., "Preventing contrast medium-induced acute kidney injury," European Radiology, vol. 28, no. 12, pp. 5384-5395, 2018.

[5] M. Roffi, C. Patrono, J.-P. Collet et al., “2015 ESC Guidelines for the management of acute coronary syndromes in patients presenting without persistent ST-segment elevation," European Heart Journal, vol. 37, no. 3, pp. 267-315, 2015.

[6] W.-J. Bei, K. Wang, H.-L. Li et al., "Safe hydration volume to prevent contrast-induced acute kidney injury and worsening heart failure in patients with heart failure and preserved ejection fraction after cardiac catheterization," Journal of Cardiovascular Pharmacology, vol. 70, no. 3, pp. 168-175, 2017.

[7] G. Qian, Z. Fu, J. Guo, F. Cao, and Y. Chen, "Prevention of contrast-induced nephropathy by central venous pressureguided fluid administration in chronic kidney disease and congestive heart failure patients," JACC: Cardiovascular Interventions, vol. 9, no. 1, pp. 89-96, 2016.

[8] S. A. Silver, P. M. Shah, G. M. Chertow, S. Harel, R. Wald, and Z. Harel, "Risk prediction models for contrast induced nephropathy: systematic review," Bmj, vol. 351, p. h4395, 2015.

[9] R. Mehran, E. Aymong, E. Nikolsky et al., "A simple risk score for prediction of contrast-induced nephropathy after percutaneous coronary interventionDevelopment and initial validation," Journal of the American College of Cardiology, vol. 44, no. 7, pp. 1393-1399, 2004. 
[10] J. J. V. McMurray, S. D. Solomon, S. E. Inzucchi et al., "Dapagliflozin in patients with heart failure and reduced ejection fraction," The New England Journal of Medicine, vol. 381, no. 21, pp. 1995-2008, 2019.

[11] R. S. Wright, J. L. Anderson, C. D. Adams et al., "2011 ACCF/ AHA focused update of the guidelines for the management of patients with unstable Angina/non-ST-elevation myocardial infarction (updating the 2007 guideline)," Journal of the American College of Cardiology, vol. 57, no. 19, pp. 1920-1959, 2011.

[12] D. W. Allen, B. Ma, K. C. Leung et al., "Risk prediction models for contrast-induced acute kidney injury accompanying cardiac catheterization: systematic review and meta-analysis," Canadian Journal of Cardiology, vol. 33, no. 6, pp. 724-736, 2017.

[13] Y. Liu, J. Y. Chen, N. Tan et al., "Safe limits of contrast vary with hydration volume for prevention of contrast-induced nephropathy after coronary angiography among patients with a relatively low risk of contrast-induced nephropathy," Circulation: Cardiovascular Interventions, vol. 8, no. 6, 2015.

[14] K. Thygesen, J. S. Alpert, A. S. Jaffe et al., "Third universal definition of myocardial infarction," Journal of the American College of Cardiology, vol. 60, no. 16, pp. 1581-1598, 2012.

[15] G. Chi, C. M. Gibson, Y. Liu et al., "Inverse relationship of serum albumin to the risk of venous thromboembolism among acutely ill hospitalized patients: analysis from the APEX trial," American Journal of Hematology, vol. 94, no. 1, pp. 21-28, 2019.

[16] P. E. Stevens and A. Levin, "Evaluation and management of chronic kidney disease: synopsis of the kidney disease: improving global outcomes 2012 clinical practice guideline," Annals of Internal Medicine, vol. 158, no. 11, pp. 825-830, 2013.

[17] M. T. Roe, A. Y. Chen, L. Thomas et al., "Predicting long-term mortality in older patients after non-ST-segment elevation myocardial infarction: the CRUSADE long-term mortality model and risk score," American Heart Journal, vol. 162, no. 5, pp. 875-883, 2011, e871.

[18] E. W. Tang, C.-K. Wong, and P. Herbison, "Global Registry of Acute Coronary Events (GRACE) hospital discharge risk score accurately predicts long-term mortality post acute coronary syndrome," American Heart Journal, vol. 153, no. 1, pp. 29-35, 2007.

[19] E. W. Steyerberg and Y. Vergouwe, "Towards better clinical prediction models: seven steps for development and an ABCD for validation," European Heart Journal, vol. 35, no. 29, pp. 1925-1931, 2014.

[20] Z. Lei, J. Li, D. Wu et al., "Nomogram for preoperative estimation of microvascular invasion risk in hepatitis B virusrelated hepatocellular carcinoma within the milan criteria," JAMA Surgery, vol. 151, no. 4, pp. 356-363, 2016.

[21] Z. You, K. Lin, W. Zheng et al., "Association of prealbumin levels with contrast-induced acute kidney injury in elderly patients with elective percutaneous coronary intervention," Clinical Interventions in Aging, vol. 13, pp. 641-649, 2018.

[22] Y.-M. Gao, D. Li, H. Cheng, and Y.-P. Chen, "Derivation and validation of a risk score for contrast-induced nephropathy after cardiac catheterization in Chinese patients," Clinical and Experimental Nephrology, vol. 18, no. 6, pp. 892-898, 2014.

[23] E. C. Nijssen, M. A. P. Vermeeren, M. M. A. Janssen et al., "Contrast material-induced nephropathy in the era of hydration," Radiology, vol. 265, no. 3, pp. 978-979, 2012.

[24] M. Almendarez, H. S. Gurm, J. Mariani Jr. et al., "Procedural strategies to reduce the incidence of contrast-induced acute kidney injury during percutaneous coronary intervention," JACC: Cardiovascular Interventions, vol. 12, no. 19, pp. 1877-1888, 2019.

[25] S. Kim, J. Sung, W. Chul Kang et al., "Increased plasma osmolar gap is predictive of contrast-induced acute kidney injury," The Tohoku Journal of Experimental Medicine, vol. 228, no. 2, pp. 109-117, 2012.

[26] G. Dangas, I. Iakovou, E. Nikolsky et al., "Contrast-induced nephropathy after percutaneous coronary interventions in relation to chronic kidney disease and hemodynamic variables," The American Journal of Cardiology, vol. 95, no. 1, pp. 13-19, 2005.

[27] G. Andò, G. Morabito, C. de Gregorio, O. Trio, F. Saporito, and G. Oreto, "Age, glomerular filtration rate, ejection fraction, and the AGEF score predict contrast-induced nephropathy in patients with acute myocardial infarction undergoing primary percutaneous coronary intervention," Catheterization and Cardiovascular Interventions Official Journal of the Society for Cardiac Angiography and Interventions, vol. 82, no. 6, pp. 878-885.

[28] P. Aubry, G. Brillet, L. Catella, A. Schmidt, and S. Bénard, "Outcomes, risk factors and health burden of contrast-induced acute kidney injury: an observational study of one million hospitalizations with image-guided cardiovascular procedures," BMC Nephrology, vol. 17, no. 1, p. 167, 2016.

[29] P. C. Fan, T. H. Chen, C. C. Lee, T. Y. Tsai, Y. C. Chen, and C. H. Chang, "ADVANCIS score predicts acute kidney injury after percutaneous coronary intervention for acute coronary syndrome," International Journal of Medical Sciences, vol. 15, no. 5, pp. 528-535, 2018.

[30] U. Sadat, A. Usman, J. R. Boyle, P. D. Hayes, and R. J. Solomon, "Contrast medium-induced acute kidney injury," Cardiorenal Medicine, vol. 5, no. 3, pp. 219-228, 2015.

[31] Y.-C. Lee, C.-C. Hsieh, T.-T. Chang, and C.-Y. Li, "Contrastinduced acute kidney injury among patients with chronic kidney disease undergoing imaging studies: a meta-analysis," American Journal of Roentgenology, vol. 213, no. 4, pp. 728735, 2019.

[32] B. Pandya, J. M. Chalhoub, V. Parikh et al., "Contrast media use in patients with chronic kidney disease undergoing coronary angiography: a systematic review and meta-analysis of randomized trials," International Journal of Cardiology, vol. 228, pp. 137-144, 2017.

[33] A. F. Kopp, K. J. Mortele, Y. D. Cho, P. Palkowitsch, M. A. Bettmann, and C. D. Claussen, "Prevalence of acute reactions to iopromide: postmarketing surveillance study of 74,717 patients," Acta Radiologica, vol. 49, no. 8, pp. 902-911, 2008.

[34] J. Q. Yang, P. Ran, J. Y. Chen et al., "Development of contrastinduced acute kidney injury after elective contrast media exposure in patients with type 2 diabetes mellitus: effect of albuminuria," PLoS One, vol. 9, no. 9, Article ID e106454, 2014.

[35] S. Akyuz, T. Kemaloglu Oz, S. Altay et al., “Association between glycosylated haemoglobin level and contrast-induced acute kidney injury in patients with type 2 diabetes mellitus," Cardiorenal Medicine, vol. 4, no. 2, pp. 95-102, 2014.

[36] L. Lei, Y. Xue, Z. Guo et al., "Population attributable risk estimates of risk factors for contrast-induced acute kidney injury following coronary angiography: a cohort study," BMC Cardiovascular Disorders, vol. 20, no. 1, p. 289, 2020.

[37] F. Deng, M. Peng, J. Li, Y. Chen, B. Zhang, and S. Zhao, "Nomogram to predict the risk of septic acute kidney injury in 
the first $24 \mathrm{~h}$ of admission: an analysis of intensive care unit data," Renal Failure, vol. 42, no. 1, pp. 428-436, 2020.

[38] L. Xu, C. Li, L. Zhao et al., "Acute kidney injury after nephrectomy: a new nomogram to predict postoperative renal function," BMC Nephrology, vol. 21, no. 1, p. 181, 2020.

[39] L. Lei, Y. Xue, Z. Guo et al., "Nomogram for contrast-induced acute kidney injury in patients with chronic kidney disease undergoing coronary angiography in China: a cohort study," BMJ Open, vol. 10, no. 5, Article ID e037256, 2020. 